\title{
Financial management efficiency performance of insurers and takaful operators in Malaysia
}

\begin{abstract}
This study is aimed to assess the financial management efficiency of each insurer/takaful operator in both conventional and takaful industry. We also identify the operating system that is more efficient among the conventional and takaful system which involves 20 firms. The results from the slack-based measure (SBM) - data envelopment analysis (DEA) implied that the average insurers/takaful operators have to improve about $20 \%$ if it were to perform the best financial management practice. Clearly, the inefficiency in both functions of financial management is caused by both the input and output dimensions. This study also revealed that takaful operators exhibit a relatively more efficient financial management in terms of risk management than conventional insurers. However, it cannot be confirmed whether one type of operating system is better than the other system in terms of investment management, as the result is mixed.
\end{abstract}

Keyword: Takaful operator; Conventional insurer; Financial management; Efficiency; Slack based measure ï data envelopment analysis 